www.jmscr.igmpublication.org Impact Factor 5.244

Index Copernicus Value: 83.27

ISSN (e)-2347-176x ISSN (p) 2455-0450

crossref DOI: _https://dx.doi.org/10.18535/jmscr/v4i10.80

Journal Of Medical Science And Clinical Research

IGM Publication

An Official Publication of IGM Publication

\title{
Effectiveness of Transcutaneous Electric Nerve Stimulation on Post Operative Pain
}

\section{Authors \\ Dr Saurabh Mahamia, Dr Suresh Naik, Dr Nagendra Yadav, Dr (Brig) KVS Rana, Dr Abhishek Singh, Dr Shamshad Ahmed}

Department of Surgery, Dr D Y Patil Pune

Email: poppy.abhishek@gmail.com, Mobile: 8806444830

\begin{abstract}
Introduction: The management of post-operative pain continues to be a significant aspect of a post-operative patient care in surgical practice. TENS is a simple, non-invasive analgesia technique which is used in health care settings, due to its ease of administration, rapid onset, it is cheaper compared to long term use of drugs, analgesics \& also few side effects/ drug interactions. The method being non-toxic and non-invasive, the physiological \& psychological dependence does not develop with its use. The advantage of TENS is that the problems of respiratory depression, sedation, hypotension seen with narcotics are avoided \& reduced. This study is being undertaken to study the efficacy of TENS in management of post operative pain.

Aims \& Objectives:

1. To assess the effectiveness of TENS in the management of post-operative pain.

2. To determine the role of TENS in reducing the incidence of postoperative pain associated complications.

3. To compare the effectiveness of TENS with postoperative analgesic group and patients receiving both TENS and analgesia.

Materials \& Methods: The study included 120 patients who underwent surgeries like Appendicectomy, hernioplasty and cholecystectomy and were divided in three groups. The detailed case history of one hundred twenty cases was recorded, clinical examination and investigations carried out. Post-operative pain was assessed with visual analogue score and functional activity score from POD 1 to POD 7 and associated postoperative complications. All these factors were used to monitor the progress and assess the recovery of the patient. Depending on use of TENS or analgesics or both and divided in groups $A, B$ and $C$.

Conclusions: TENS shows a very promising effect in controlling the postoperative pain, there was a significant reduction in pain in Hernioplasty and Appendicectomy surgeries but minimal in Cholecystectomy. TENS also shows that side effects of analgesics drugs are reduced to the minimal level.

Key Words:- TENS, Post-operative Analgesia, functional activity score.
\end{abstract}

\section{INTRODUCTION}

Pain defined as a complex, unpleasant perception associated with emotional and sensory experience with actual and potential tissue injury as disease process. ${ }^{(1)}$ (According to international association of study of pain- IASP).
Post-operative pain is a complex response to tissue trauma during surgery that stimulates hypersensitivity of the central nervous system. The result is pain in areas not directly affected by the surgical procedure. ${ }^{(2)}$ 
However the effective doses of narcotic agents or their synthetic derivatives used for modern medical \& surgical therapeutics, apart from producing analgesia, also produce unwanted side effects. $^{(3)}$

\section{TRANSCUTANEOUS ELECTRIC NERVE} STIMULATION [TENS] is defined as the application of low frequency current in the form of pulsed rectangular currents through surface electrodes on the patient's skin to reduce pain. ${ }^{(4)}$

TENS is a simple, cheap, non-toxic and noninvasive analgesia technique which is used in health care settings, with few side effects/drug interactions. The physiological \& psychological dependence like respiratory depression, sedation and physical dependence seen with Diclofenac Sodium does not develop with its use. ${ }^{(4)}$

It's use in the immediate post-operative period has been reported to be followed by a reduction in post-operative complication including atelectasis $\&$ urinary retention as well as reduction in drug requirements for pain management. ${ }^{(4)}$

There seems to be a high level of patient acceptance \& some reports have noted that TENS enhances the physician-patient relationship due to the extra concern for patient welfare \& comfort that is shown when TENS is being used. ${ }^{(5)}$

The aim of this study is to evaluate the role of TENS in the management of postoperative pain.

\section{AIMS AND OBJECTIVES}

1. To assess the effectiveness of TENS in the management of post-operative pain.

2. To assess the effectiveness of TENS on post-operative functional activity.

3. To determine the role of TENS in reducing the incidence of post-operative complications.

4. To compare the effectiveness of only TENS with postoperative analgesic group with patients receiving both TENS and Analgesia.

Plan of study - A prospective simple, randomized comparative study of effectiveness of TENS in the management of post-operative pain in patients undergoing Appendicectomy, hernioplasty and cholecystectomy was carried out. Institutional Ethical Committee approval was taken before the commencement of the study. Informed consent was obtained from all the patients before inclusion in the study.

INCLUSION CRITERIA: Patients of age between 15 to 65 years undergoing appendicectomy, cholecystectomy and hernioplasty.

EXCLUSION CRITERIA: Age less than 15yrs of Age, patients with cardiac pacemakers, patients suffering from epilepsy, woman in the first trimester of pregnancy, elderly $>65$ years.

Sample size was 120 cases for this study.

A total 120 patients of Appendicectomy, Hernioplasty and Cholecystectomy who had fulfilled the inclusions and exclusion criteria were included in the study.

The study population was divided into three groups:

Group A: Postoperative analgesia provided by TENS only.

Group B: Postoperative analgesia provided by Analgesics drugs. (CONTROL GROUP)

Group C: Postoperative analgesia provided by both TENS and Analgesics drugs.

Each group was further subdivided into following subgroups depend upon the type of surgery.

Group A1: TENS group patients undergoing Appendicectomy (14cases)

Group A2: TENS group patients undergoing Hernioplasty (13cases)

Group A3: TENS group patients undergoing Cholecystectomy (13cases)

Group B1: Analgesics group patients undergoing Appendicectomy (13cases)

Group B2: Analgesics group patients undergoing Hernioplasty (14cases)

Group B3: Analgesics group patients undergoing Cholecystectomy (13cases)

Group C1: TENS with analgesics group Patients undergoing Appendicectomy (13cases)

Group C2: TENS with analgesics group Patients undergoing hernioplasty (13cases) 
Group C3: TENS with analgesics group Patients undergoing Cholecystectomy (14cases)

\section{OPERATIVE PROTOCOL}

Patients were initially examined in the OPD and surgical ward all routine investigations were sent and assessment of fitness was carried out by anaesthetist. First three patients were selected by the lottery and randomized selections of patients were done in three study groups prior to the operative procedures. Patients were assessed preoperatively as per the proforma attached (Annexure II) and evaluated as per master chart.

\section{METHOD}

All patients were explained the procedure in details to allay any anxiety, how to apply TENS at the operated site, how to control the intensity of the TENS machine and effects, how to assess pain and record it in pain chart. They were made aware of the local skin irritation due to heat and allergy to adhesive tapes used.

Description of TENS: Conventional TENS (Jai Surgical's Pvt Ltd.) of Cost rupees 2000 only, with stimulation frequency (40-150HZ) of Low intensity with the current set between 10-30 MA with Carbon impregnated 4 rubber electrodes. Aqua Sonic Gel was used for local application.

Application of TENS: Two sterile electrodes (first unit channel were placed on one side of incision and two other (second unit channel), on the other side. The electrodes were placed $2 \mathrm{~cm}$ away from the suture line. Frequency was fixed up to $50 \mathrm{HZ}$ and intensity as per the patient tolerance $(0-60$ MA).

Description of Analgesics: Trade name:- Generic name:- Diclofenac Sodium, intramuscular Injection Voveran (3 ml Ampoule of Strength 25 $\mathrm{mg} / \mathrm{ml}$ ) and Tablet Voveran SR (75 mg Tablet).

Doses and Route of administration: $75 \mathrm{mg}$ deep Intramuscular Inj. Diclofenac Sodium 8 hrly on POD1 to POD2 followed by Tab. Diclofenac Sodium $75 \mathrm{mg}$ was given on POD3 to POD4. Subsequently Tab. Diclofenac Sodium $75 \mathrm{mg}$ was given as per the requirement.

\section{POSTOPERATIVE PROTOCOL}

Group A: Pain was assessed on patients with TENS only every 4 hrly on POD1 and POD 2. From POD3 to POD7 pain was assessed every 8 hrly. No analgesics were used.

Group B: Pain was assessed on patients with analgesia only every 4 hrly on POD1 and POD2. From POD3 to POD7 pain was assessed every 8 hrly. No TENS was used.

Group C: Pain was assessed on patients with TENS and Analgesics every 4 hrly on POD1 and POD2. From POD3 to POD7 pain was assessed every 8 hrly. Both TENS and analgesics were used

\section{ASSESSMENT}

Pain was assessed every 4 hrly from POD1 and POD2 and every 8 hrly from POD3 to POD7 by Visual Analogue Scale (figure 5)

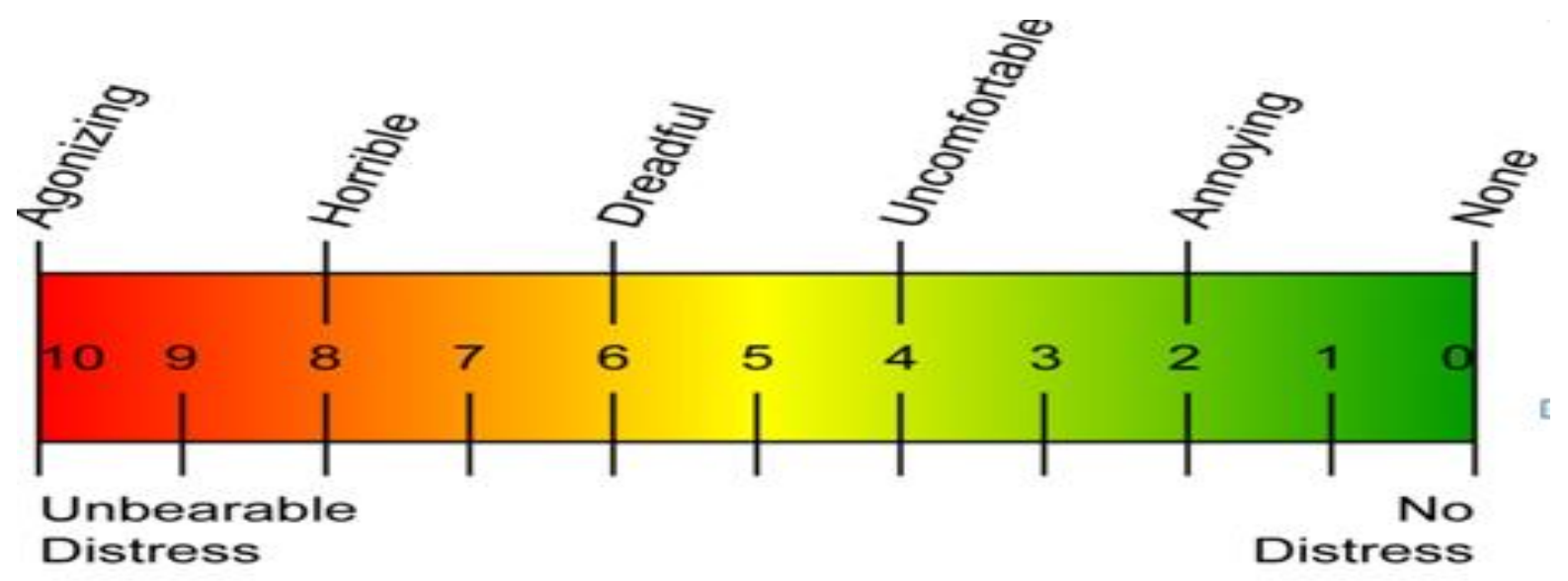

Figure . Visual Analogue Scale 
Functional Activity Score:-an activity related score in which patient were asked to perform an activity related ie. Deep breath/ Cough/ Ambulation.

We Observed patient functional activity every 4 hrly from POD1 and POD2 and 8 hrly from POD3 to POD7 for the chosen activity and scored as A, $\mathrm{B}$ or $\mathrm{C}$.

A - No limitation meaning the patient's activity is unrestricted by pain.

B - Mild limitation means the patient's activity is mild to moderately restricted by pain.

C - Severe limitation means the patient ability to perform the activity is severely limited by pain.
Assessment of postoperative complications: The post-operative complications ie. Nausea, vomiting, urinary retention, skin allergy/ irritation and respiratory complications were observed from POD1 to POD7.

Follow Up:-Assessment was done with regard to pain score, functional activity score and postoperative complications during first seven days and at the time of discharge.

Data Analysis: All collected data was analyzed by the resident and statistical analysis was done using Annova and Chi-Square test as per sample size.

\section{Comparison of VAS In Study Groups:}

\section{Table 1}

\begin{tabular}{|c|c|c|c|}
\hline \multirow[t]{3}{*}{ Group } & & VAS score & \multirow[t]{3}{*}{ P Value } \\
\hline & & POD1 & \\
\hline & & Mean \pm SD & \\
\hline \multirow[t]{3}{*}{ Appendicectomy } & $\mathrm{A} 1(\mathrm{n}=14)$ & $4.92 \pm 0.27$ & \multirow[t]{3}{*}{$<0.0001$} \\
\hline & $\mathrm{B} 1(\mathrm{n}=13)$ & $5 \pm 0$ & \\
\hline & $\mathrm{C} 1(\mathrm{n}=13)$ & $4 \pm 0$ & \\
\hline \multirow[t]{3}{*}{ Hernioplasty } & $\mathrm{A} 2(\mathrm{n}=13)$ & $5 \pm 0$ & \multirow[t]{3}{*}{ - } \\
\hline & $\mathrm{B} 2(\mathrm{n}=14)$ & $5 \pm 0$ & \\
\hline & $\mathrm{C} 2(\mathrm{n}=13)$ & $4 \pm 0$ & \\
\hline \multirow[t]{3}{*}{ Cholecystectomy } & A3 $(n=13)$ & $6 \pm 0$ & \multirow[t]{3}{*}{-} \\
\hline & B3 $(n=13)$ & $5 \pm 0$ & \\
\hline & C3 $(n=14)$ & $4 \pm 0$ & \\
\hline
\end{tabular}

Table 2

\begin{tabular}{|c|c|c|c|c|}
\hline VAS score & \multirow[t]{3}{*}{ P Value } & VAS score & \multirow[t]{3}{*}{ P Value } & VAS score \\
\hline POD2 & & POD3 & & POD7 \\
\hline Mean \pm SD & & Mean \pm SD & & Mean \pm SD \\
\hline $4 \pm 0$ & \multirow[t]{3}{*}{$<0.0001$} & $2.93 \pm 0.27$ & \multirow[t]{3}{*}{$<0.0001$} & $0 \pm 0$ \\
\hline $4 \pm 0$ & & $3 \pm 0$ & & $0 \pm 0$ \\
\hline $3.08 \pm 0.28$ & & $2 \pm 0$ & & $0 \pm 0$ \\
\hline $4 \pm 0$ & \multirow[t]{3}{*}{-} & $3 \pm 0$ & \multirow[t]{3}{*}{-} & $0 \pm 0$ \\
\hline $4 \pm 0$ & & $3 \pm 0$ & & $0 \pm 0$ \\
\hline $3 \pm 0$ & & $2 \pm 0$ & & $0 \pm 0$ \\
\hline $5 \pm 0$ & \multirow[t]{3}{*}{-} & $4 \pm 0$ & \multirow[t]{3}{*}{-} & $0 \pm 0$ \\
\hline $5 \pm 0$ & & $4 \pm 0$ & & $0 \pm 0$ \\
\hline $4 \pm 0$ & & $3 \pm 0$ & & $0 \pm 0$ \\
\hline
\end{tabular}




\section{JMSCR Vol||04||Issue ||10||Page 13290-13298||October}

VAS was low in Group C as compared to Group $\mathrm{A} \& \mathrm{~B}$ on POD3. On POD1 \& POD2 VAS was same in Group A\& Group B. There was a significant reduction in VAS in Group A from POD2 to POD7 in all groups.

\section{Figure1}

Bar diagram showing day wise comparison of VAS score in study groups

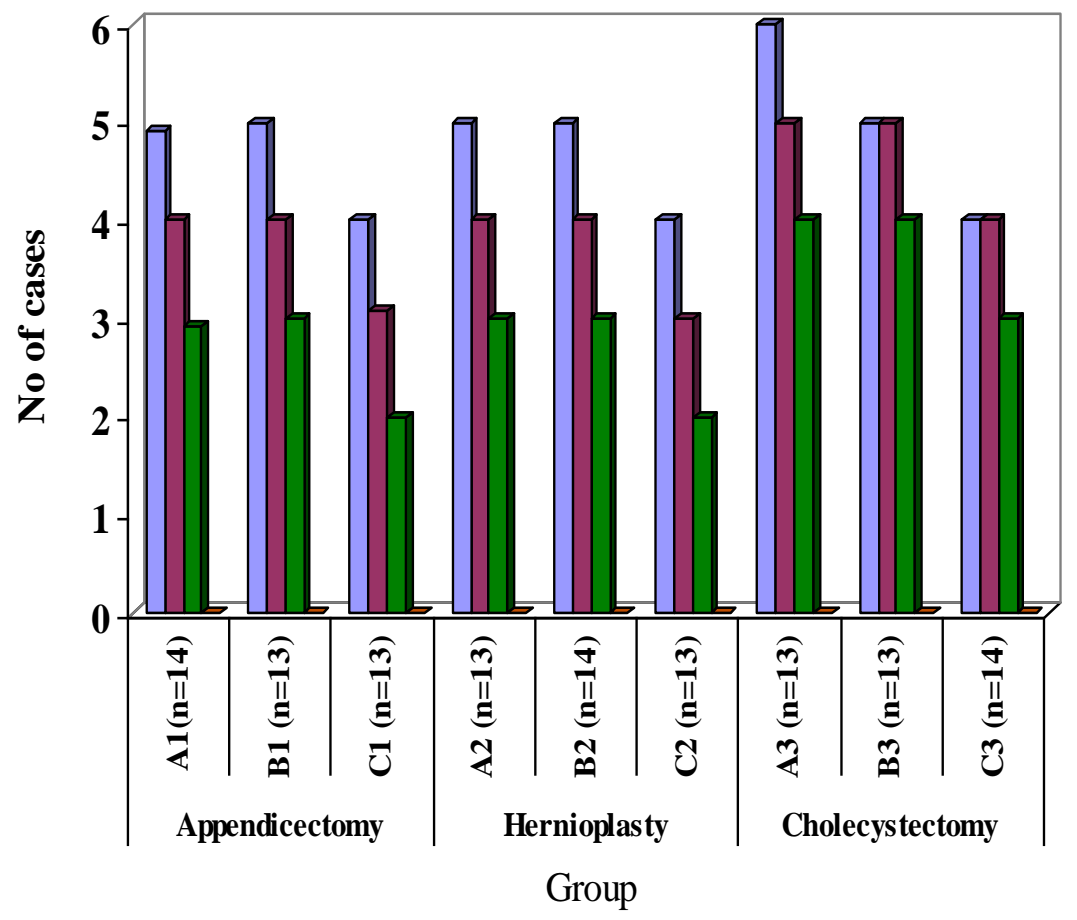

$\square$ VAS score POD1 $\square$ VAS score POD2 $\square$ VAS score POD3 $\square$ VAS score POD7

\section{Comparison of FAS in Study Groups: Table 3}

\begin{tabular}{|c|c|c|c|c|c|}
\hline \multirow[t]{3}{*}{ Group } & & \multicolumn{3}{|c|}{ FAS } & \multirow[t]{3}{*}{ P Value } \\
\hline & & \multicolumn{3}{|c|}{ POD1 } & \\
\hline & & $\mathbf{A}$ & $\mathbf{B}$ & $\mathbf{C}$ & \\
\hline \multirow[t]{3}{*}{ Appendicectomy } & $\mathrm{A} 1(\mathrm{n}=14)$ & 0 & 14 & 0 & \multirow[t]{3}{*}{$<0.0001$} \\
\hline & B1 $(n=13)$ & 0 & 13 & 0 & \\
\hline & $\mathrm{C} 1(\mathrm{n}=13)$ & 0 & 0 & 13 & \\
\hline \multirow[t]{3}{*}{ Hernioplasty } & A2 $(n=13)$ & 0 & 13 & 0 & \multirow[t]{3}{*}{$<0.0001$} \\
\hline & $\mathrm{B} 2(\mathrm{n}=14)$ & 0 & 14 & 0 & \\
\hline & $\mathrm{C} 2(\mathrm{n}=13)$ & 0 & 0 & 13 & \\
\hline \multirow[t]{3}{*}{ Cholecystectomy } & A3 $(n=13)$ & 0 & 13 & 0 & \multirow[t]{3}{*}{$\begin{array}{lll}- & - \\
-1\end{array}$} \\
\hline & B3 $(n=13)$ & 0 & 13 & 0 & \\
\hline & C3 $(n=14)$ & 0 & 14 & 0 & \\
\hline
\end{tabular}




\section{JMSCR Vol||04||Issue || 10||$P a g e ~ 13290-13298 \|$ October}

Table 4

\begin{tabular}{|c|c|c|c|c|c|c|c|c|c|c|}
\hline \multirow{2}{*}{\multicolumn{3}{|c|}{$\begin{array}{c}\text { FAS } \\
\text { POD2 }\end{array}$}} & \multirow[t]{3}{*}{ P Value } & \multirow{2}{*}{\multicolumn{3}{|c|}{$\begin{array}{c}\text { FAS } \\
\text { POD3 }\end{array}$}} & \multirow[t]{3}{*}{ P Value } & \multirow{2}{*}{\multicolumn{3}{|c|}{$\begin{array}{c}\text { FAS } \\
\text { POD7 }\end{array}$}} \\
\hline & & & & & & & & & & \\
\hline$\overline{\mathbf{A}}$ & B & $\mathbf{C}$ & & $\mathbf{A}$ & B & $\mathrm{C}$ & & $\mathbf{A}$ & B & $\mathbf{C}$ \\
\hline 0 & 14 & 0 & \multirow[t]{3}{*}{ - } & 14 & 0 & 0 & \multirow[t]{3}{*}{$<0.0001$} & 14 & 0 & 0 \\
\hline 0 & 13 & 0 & & 13 & 0 & 0 & & 13 & 0 & 0 \\
\hline 0 & 13 & 0 & & 0 & 13 & 0 & & 13 & 0 & 0 \\
\hline 0 & 13 & 0 & \multirow[t]{3}{*}{$<0.0001$} & 13 & 0 & 0 & \multirow[t]{3}{*}{$<0.0001$} & 13 & 0 & 0 \\
\hline 0 & 14 & 0 & & 14 & 0 & 0 & & 14 & 0 & 0 \\
\hline 0 & 0 & 13 & & 0 & 13 & 0 & & 13 & 0 & 0 \\
\hline 0 & 13 & 0 & \multirow[t]{3}{*}{ - } & 13 & 0 & 0 & \multirow[t]{3}{*}{$<0.0001$} & 13 & 0 & 0 \\
\hline 0 & 13 & 0 & & 13 & 0 & 0 & & 13 & 0 & 0 \\
\hline 0 & 14 & 0 & & 0 & 14 & 0 & & 14 & 0 & 0 \\
\hline
\end{tabular}

FAS were better in Group C as compare to Group A\& B on POD3. FAS was better in group A in Appendicectomy and Hernioplasty surgery as compare to group B on POD1. In group A FAS was more in cholecystectomy surgery as compare to group B \& Group C on POD1. There was significant reduction in FAS in Group A from POD2 to POD7.

Figure 2

Bar diagram showing day wise comparison of FAS score in study groups

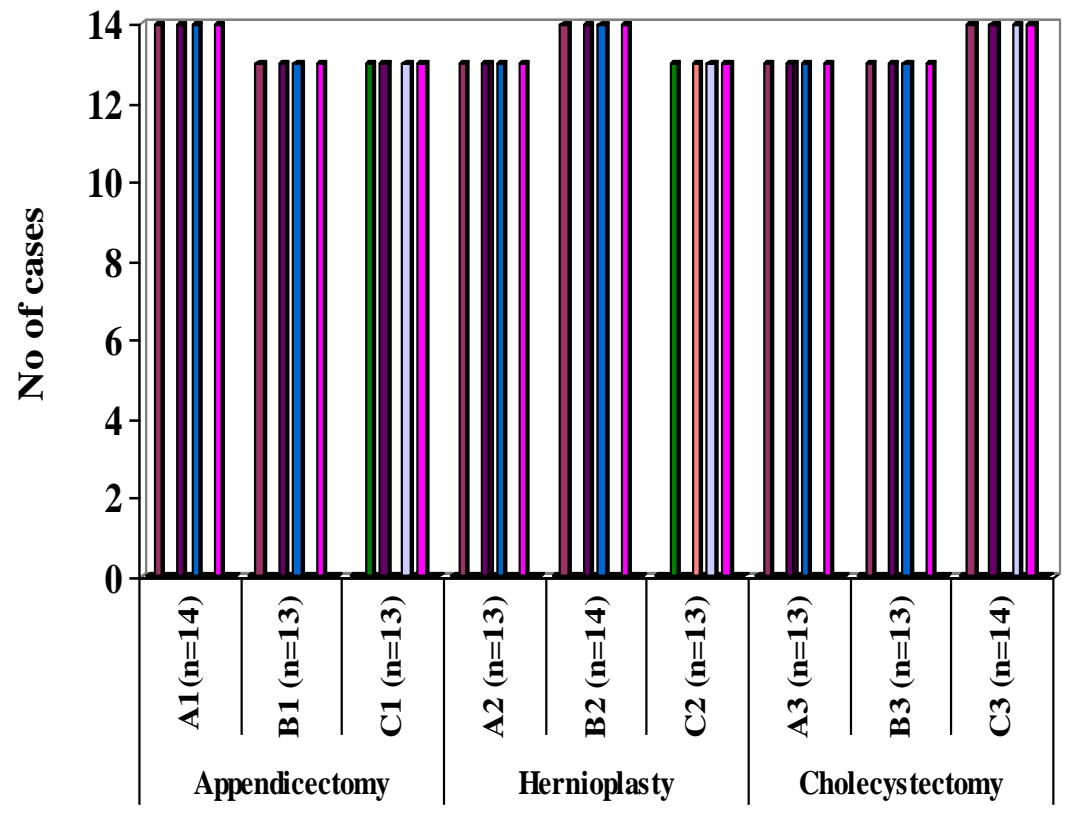

Group

口FASPOD1 A $\quad$ FAS POD1 B $\quad$ FASPOD1C $\quad$ FASPOD2 A $\quad$ FASPOD2 B $\quad$ FASPOD2C

$\square$ FAS POD3 A $\quad$ FAS POD3 B $\quad$ FAS POD3C $\quad$ FAS POD7 A $\quad$ FASPOD7 B $\quad$ FAS POD7C 


\section{DISCUSSION}

Pain is considered as the fifth vital sign along with pulse, blood pressure, temperature and respiration. Postoperative pain increases post-surgical complica-tions, raises the cost and interferes with recovery and return to normal activities of daily living. Management of postsurgical pain fasten recovery and aids in healing process.

In this study the age of the patients in all the groups were comparable. The mean age in Appendicectomy case was 25 years, in hernioplasty cases was 45 years and in cholecystectomy cases was 42 years. There was no statically significant age difference seen in all three Groups. Out of 120 patients 78 were males and 42 females

\section{POSTOPERATIVE PAIN ASSESSMENT BY VISUAL ANALOGUE SCALE}

Appendicectomy cases: In this study VAS was better in Group C as compare to Group A\& B from POD1 to POD3. In Group A \& B VAS was same on POD1, But There was significant decrease in postoperative pain in Group A compare to Group B from POD2 to POD7 This study was comparable with study done by $\mathbf{S}$. N. Yadav, I.G.Conn et al of 30 patients underwent Appendicectomy showed that there was significant decrease in pain severity and analgesic intake when TENS compared with the Analgesic Group. ${ }^{(6)}$

Hernioplasty cases: In this study VAS was better in Group C as compare to Group A\& B from POD1 to POD3. In Group A \& B VAS was same on POD1, But There was significant decrease in postoperative pain in Group A compare to Group $\mathrm{B}$ from POD1 to POD7 $(\mathrm{P}<0.001)$.This study was comparable with study done by De Santana et al of 40 patients who underwent unilateral inguinal herniorrhaphy in which Pain was assessed before and after each application of TENS and 8 and 24 hours after surgery. In the group treated with active TENS, pain intensity was significantly lower 2 hours $(P=.028), 4$ hours $(P=.022), 8$ hours $(P=.006)$, and 24 hours $(P=.001)$ after the surgery. Active TENS also decreased analgesic requirements in the postoperative period $(P=.001){ }^{(7)}$

Cholecystectomy cases: In this study VAS was better in Group C as compare to Group A\& B from POD1 to POD3. On POD1 the VAS was high in Group A compare to Group B, But there was significant reduction in postoperative pain in Group A from POD2 to POD7 ( $<<0.001)$. This study was comparable with study done by Dougal T sim et al of30patients undergoing elective cholecystectomy. The effectiveness of TENS was evaluated by measuring the analgesic effects on the first, second and fifth post-operative days using linear analogue pain scales. Analysis of subjective pain measurements revealed similar pain scores for both groups on all but the second post-operative day where a significant decrease ( $\mathrm{p}$ $<0.05$ ) was found for both rest pain and deep breathing pain scores for the TENS group. Patients within the TENS group also recorded significantly less pain on fifth post-operative day. ${ }^{(8)}$

\section{POSTOPERATIVE ACTIVITY ASSESSME- NT BY FUNCTIONAL ACTIVITY SCORE:}

Appendicectomy cases: In this Study FAS was better in Group C compare to Group A\& B upto POD3. In Group A FAS was better as compare to Group B upto POD7. On POD7 FAS was equal in all three Groups

Hernioplasty cases: In this Study FAS was better in group C compare to Group A\& B upto POD3. In group A FAS was better as compare to Group B up to POD7. On POD7 FAS was equal in all three groups

Cholecystectomy cases: In this Study FAS was better in Group C compare to Group A\& B upto POD3. FAS were better in Group B than A on POD1. There was significant improvement in FAS in Group A from POD2 to POD7.This study was comparable with study done by Dougal $\mathbf{T}$ sim et al of 30 patients undergoing elective cholecystectomy. The effectiveness of TENS was evaluated by measuring both the analgesic and pulmonary effects on the first, second and fifth post-operative days in which measured at rest, following deep 
breathing, and following coughing, using linear analogue pain scales. ${ }^{(8)}$

Post-operative complications- The incidence of nausea was less in group A. Vomiting,urinary retention and respiratory complications were also less as compared to group B and C. No incidence of skin irritation was noted in all Groups

In a study by Eduardo Zarate et al of 221 outpatients undergoing laparoscopic cholecystectomy showed that TENS was associated with a significantly decreased incidence of moderate to severe Nausea $(\mathrm{P}<0.05)$. TENS was also associated with a larger proportion of patients free from moderate to severe nausea symptoms; $\mathrm{P}<$ 0.05).TENS reduced postoperative nausea and vomiting, in outpatients undergoing laparoscopic cholecystectomy procedures. ${ }^{(9)}$. In the study by $\mathbf{C}$ G Morran et al to assess TENS in management of postoperative pain and its effect on Postoperative Pulmonary function and Respiratory complications. He noted that pain score over the three days of study showed that both groups experienced similar degrees of pain on comparison of TENS with analgesics. There were no significant differences in postoperative pulmonary function and incidence of respiratory complications was similar in both groups. ${ }^{(10)}$

In this study of 120 patients who underwent Appendicectomy, Hernioplasty, Cholecystectomy surgery were divided in three Groups as: A) TENS only, B) Analgesics only, C) TENS with analgesics.

The outcome of the study shows that TENS with analgesics Group was the best group compare to other two Groups. VAS and FAS was better in Group C as compare to Group A\& Group B. Postoperative complications were less in Group C as compare to Group A \& B.

On comparison of Group A \& B TENS was equal in Appendicectomy and hernioplasty cases on POD1. But there was a significant reduction in VAS \& FAS in Group A from POD2 To POD7. Tens was not so effective in Cholecystectomy cases on POD1, But there was significant reduction in VAS \& FAS from POD2 to POD7.
The incidence of postoperative complications was less in Group C as compare to Group A\& B. On comparison of Group A \& B TENS group showed less complications as compare to analgesics and no incidence of local skin irritation.

This study shows that TENS alone is effective in postoperative pain management in Appendicectomy \& Hernioplasty cases. TENS improves Functional Activity better than analgesics group. In cholecystectomy cases TENS was not effective on POD1 but there was reduction in pain from POD2 to POD7.

The incidence of postoperative complications was less in TENS Group as compare to analgesics group.

\section{LIMITATIONS}

There was no postoperative pain management team to manage postoperative pain and only the mentee was responsible for collection of data and management so the bias of the mentee in analysis of data cannot be ruled out.In this study no placebo TENS was used so patient bias after application of TENS cannot be ruled out.

Pain is a subjective feeling. The VAS is based on patients own subjective assessment which may not be correct.

\section{RECOMMENDATIONS}

Based on the multimodal protocol for postoperative pain management it is necessary to use those modalities which act on different centres by which pain can be managed more effectively with minimal complications.Co-operation of patient and family members is also important for the management of postoperative pain.As TENS is effective in Appendicectomy and hernioplasty surgery it should be incorporated in every postoperative case for the reduction of analgesic requirements and associated complications.

\section{CONCLUSIONS}

1) Management of postsurgical pain helps to improve patient's daily activities and healing process. 
2) TENS is a simple, non-invasive analgesia technique, easy to apply due to its ease of administration and rapid onset; it is cheaper compared to long term use of drugs and has few side effects/ drug interactions.

3) The results of TENS were comparable to analgesics.

4) In this study TENS was also helpful in improvement of FAS.

5) In this study TENS was helpful in minimize pain score.

6) TENS reduced postoperative complications compare to analgesics.

7) TENS alone was effective in Appendicectomy \& Hernioplasty surgery.

8) In TENS group pain score was more in cholecystectomy as compare to analgesic group.

9) TENS with analgesics group was the best group which is helpful in reduction of postoperative analgesics requirement reducing complications of analgesics.

\section{REFERENCES}

1. Pamela E Macintyre, David A Scott, Stephen A schug, Eric J Visser, Suellen M Walker. Acute pain management scientific evidence. $3^{\text {rd }}$ edi. Melbourne, Australia, Australian \& New Zealand college of Anaesthetists.2010;3:1-6.

2. Wu, Z. A. Casey. "Managing Postoperative Pain in the Opioid tolerant Patient: Careful Planning Provides Optimal Pain Control, Minimizes Problems." Journal of Critical Illness.2002;17(11):426-33.

3. Thurin E, Meehan P, Gilbert B. Treatment of pain by Transcutaneous electric nerve stimulation in general practice. Med J Aus.1980;1:70-1.

4. Mark Johnson, Pope, Mockett, Wright. Low frequency currents. $1^{\text {st }}$ edi. Academic press, San Diego USA. Elsevier Inc. 2004;1:299-306.
5. Frederick L. Schomburg, Sue ann Carterbaker: Transcutaneous electrical nerve stimulation for postlaparotomy pain. American physical therapy association 1983;6:188-93.

6. I.G. CONN, A. H. Marshall, S. N. Yadav, J. C. Daly, and M. Jaffer: Transcutaneous electric nerve stimulation following Appendicectomy: the placebo effect. Ann RColl Surg Engl.1986;68(4):191-2.

7. De Santana, DaniloRiberio, A Kathleen A.A Sluka, Ranaldo Q.A Gurgel. Hypoalgesic effect of the transcutaneous electrical nerve stimulation following inguinal herniorrhaphy: A Randomized, controlled trial.2008;9:623-9.

8. Dougal T Sim. Effectiveness of Transcutaneous Electrical Nerve Stimulation Following Cholecystectomy. American Journal of physiotherapy.1991;77(10):715-22.

9. Eduardo Zarate, Melinda Mingus, Paul F. White, JEN W. Chiu. The Use of Transcutaneous Acupoint Electrical Stimulation for Preventing Nausea and Vomiting After Laparoscopic Surgery: International Anaesthesia Research Society. 2001; 92:623-35.

10. R. J. Cuschieri, C. G. Morran, C. S. McArdle: Transcutaneous electrical stimulation for postoperative pain. Ann R Coll Surg Engl.1985;67(2):127-9. 